\title{
UV emission line shifts of symbiotic binaries
}

\author{
M. Friedjung ${ }^{1}$, J. Mikołajewska ${ }^{2}$, A. Zajczyk ${ }^{3}$, and M. Eriksson ${ }^{4}$ \\ 1 Institut d'Astrophysique de Paris -UMR 7095, CNRS/Université Pierre et Marie Curie, 98 bis Boulevard Arago, 75014 Paris, France \\ e-mail: fried@iap.fr \\ 2 Nicolaus Copernicus Astronomical Center, Bartycka 18, 00-716 Warsaw, Poland \\ 3 Nicolaus Copernicus Astronomical Center, Rabiańska 8, 87-100 Toruń, Poland \\ 4 University College of Kalmar, 39182 Kalmar, Sweden
}

Received 9 October 2009 / Accepted 4 January 2010

\section{ABSTRACT}

\begin{abstract}
Aims. Relative and absolute emission line shifts have been previously found for symbiotic binaries, but their cause was not clear. This work aims to better understand the emission line shifts.

Methods. Positions of strong emission lines were measured on archival UV spectra of Z And, AG Dra, RW Hya, SY Mus and AX Per and relative shifts between the lines of different ions compared. Profiles of lines of RW Hya and Z And were also examined.

Results. The reality of the relative shift between resonance and intercombination lines of several times ionised atoms was clearly shown except for AG Dra. This redshift shows a well defined variation with orbital phase for Z And and RW Hya. In addition the intercombination lines from more ionised atoms and especially O IV are redshifted with respect to those from less ionised atoms. Other effects are seen in the profiles.

Conclusions. The resonance-intercombination line shift variation can be explained in quiescence by P Cygni shorter wavelength component absorption, due to the wind of the cool component, which is specially strong in inferior conjunction of this cool giant. The velocity stratification permits absorption of line emission. The relative intercombination line shifts may be connected with varying occultation of line emission near an accretion disk, which is optically thick in the continuum.
\end{abstract}

Key words. binaries: symbiotic - stars: mass-loss - accretion, accretion disks

\section{Introduction}

The variations of the radial velocities of the emission lines, formed in symbiotic systems, are not easy to interpret. The lines are emitted in moving plasma, due to winds and/or gas streams, which do not need to have the same motion as either stellar component. In addition the lines can be affected by absorption of overlying material such as the absorption of other lines of the iron forest as well as by radiative transfer effects. The line profiles are as a result not necessarily simple, affecting radial velocity measurements. The present work was undertaken in order to better understand both radial velocities and in some cases line profiles.

Among the effects previously found, there is a systematic redshift of the wavelengths of resonance emission lines of highly ionized atoms with respect to those of intercombination emission lines in the ultraviolet spectra of a number of symbiotic binaries (Friedjung et al. 1983). The former lines might be expected to be optically thick, so it appeared that such a redshift might be explainable in an expanding medium, either by the presence of absorption at the short wavelength edge of the resonance emission lines, or by a radiative transfer effect associated with the scattering of radiation in this expanding medium. The latter can occur only if the optical thickness is very large.

More recently a GHRS/HST spectrum and a number of IUE spectra of the symbiotic binary CI Cyg were studied by Mikołajewska et al. (2006). The redshift was confirmed for that binary. The resonance lines had an almost constant radial velocity during an orbital cycle, interpreted as being most probably due to the presence of a blueshifted absorption component, produced in a circum-binary region. According to the interpretation proposed, the circum-binary region appeared to be mostly expanding, while in addition, a part appeared to be contracting. Other radial velocity effects in the emission lines were also investigated and interpreted in that paper.

In the present work we examine the radial velocities of other symbiotic binaries, comparing mean radial velocities of emission lines of different ions, in order to look for optical thickness and ionisation potential dependent stratification effects. We can in this connection note that according to Nussbaumer et al. (1988), among the emission lines, which interest us C III, C IV, N III,N IV and O III are expected to be formed in the same region for a temperature of the source of ionising radiation of not less than $60000 \mathrm{~K}$ and an electron density of around $10^{9} \mathrm{~cm}^{-3}$. In addition the critical densities, where most emission of the intercombination lines is produced, are of the order of $10^{9} \mathrm{~cm}^{-3}$. This paper together with our previous paper on CI Cyg (Mikołajewska et al. 2006), completes the study of radial velocity shifts in all symbiotic binaries which have strong emission lines and for which high resolution ultraviolet spectra exist.

\section{The data}

We have used the MAST archive, containing IUE and HST spectra. Among the IUE spectra only those, having a high resolution, taken with the large aperture and the SWP camera, were analysed. HST spectra were available for AG Dra and RW Hya. This left 41 spectra of Z And, 44 spectra of AG Dra, 11 spectra of RW Hya, only 7 spectra for SY Mus and even fewer (3) for AX Per. 
Table 1. Radial velocities of the UV emission lines (in units of $\mathrm{km} \mathrm{s}^{-1}$ ) for AX Per, SY Mus and RW Hya.

\begin{tabular}{|c|c|c|c|c|c|c|c|c|c|c|c|}
\hline $\begin{array}{l}\text { MJD } \\
\text { IP }[\mathrm{eV}] \\
\end{array}$ & $\phi$ & $\begin{array}{c}\text { Si III] } \\
16.3 \\
\end{array}$ & $\begin{array}{l}\text { C III] } \\
24.4 \\
\end{array}$ & $\begin{array}{c}\mathrm{N} \text { III] } \\
29.6 \\
\end{array}$ & $\begin{array}{l}\text { O III] } \\
35.1 \\
\end{array}$ & $\begin{array}{c}\text { N IV] } \\
47.4 \\
\end{array}$ & $\begin{array}{l}\text { O IV] } \\
54.1 \\
\end{array}$ & $\begin{array}{l}\text { Si IV } \\
33.5\end{array}$ & $\begin{array}{l}\text { C IV } \\
47.5 \\
\end{array}$ & $\begin{array}{l}\mathrm{Nv} \\
77.5\end{array}$ & $\begin{array}{c}\text { He II } \\
54.4\end{array}$ \\
\hline \multicolumn{12}{|c|}{ AX Per } \\
\hline 44156 & 0.000 & -123 & -121 & $-120(2)$ & $-124(2)$ & & \multirow{3}{*}{-135} & -106 & $-105(2)$ & \multirow{3}{*}{$-111(1)$} & -113 \\
\hline 45642 & 0.183 & -137 & -131 & $-134(1)$ & $-136(5)$ & -135 & & & $-107(4)$ & & -137 \\
\hline 48226 & 0.979 & -111 & -110 & $-116(2)$ & $-111(2)$ & -111 & & $-104(2)$ & $-103(2)$ & & -113 \\
\hline \multicolumn{12}{|c|}{ SYMus } \\
\hline 44767 & 0.340 & 7 & 15 & 7 & $9(1)$ & 8 & 13 & 19(1) & $22(2)$ & $16(3)$ & 3 \\
\hline 45020 & 0.746 & & -6 & & & -10 & & & $20(1)$ & & 2 \\
\hline 48451 & 0.240 & 9 & 10 & & $2(1)$ & 7 & & & $23(2)$ & & -6 \\
\hline 48473 & 0.275 & 14 & 19 & 14 & $13(1)$ & 10 & & 26 & $27(1)$ & 28 & 3 \\
\hline 48629 & 0.525 & 16 & 16 & $16(1)$ & $16(1)$ & 13 & & $21(1)$ & $23(1)$ & $22(4)$ & 12 \\
\hline 48623 & 0.675 & 11 & 14 & 9 & 11(1) & 13 & & $18(2)$ & $23(1)$ & 21(1) & 8 \\
\hline 50187 & 0.018 & 0 & 5 & -15 & -2 & -5 & & & $23(1)$ & & -3 \\
\hline \multicolumn{12}{|c|}{ RW Hya } \\
\hline 44118 & 0.437 & 10 & 11 & 3 & $2(1)$ & -8 & $-3(2)$ & $17(1)$ & $26(3)$ & 19(1) & -26 \\
\hline 44256 & 0.809 & 7 & 6 & 7 & $6(2)$ & 8 & $32(2)$ & 24 & $36(1)$ & $32(7)$ & 34 \\
\hline 44692 & 0.987 & 14 & 23 & 17 & $18(1)$ & 24 & $47(1)$ & & $45(3)$ & & 55 \\
\hline $52012^{*}$ & 0.749 & & & & $11.9(0.5)$ & 17.7 & $35.3(0.1)$ & $29.0(1.0)$ & $40.0(1.2)$ & $46.5(0.4)$ & 40.4 \\
\hline $52016^{*}$ & 0.760 & & & & $8.7(0.3)$ & 16.1 & $34.0(0.5)$ & $29.4(0.4)$ & $38.0(1.3)$ & $48.1(0.4)$ & 39.0 \\
\hline $52020^{*}$ & 0.771 & & & & $9.5(0.8)$ & 17.1 & $35.4(0.5)$ & $30.3(0.2)$ & $39.0(0.9)$ & $50.1(0.5)$ & 40.2 \\
\hline $52024^{*}$ & 0.782 & & & & $11.9(1.1)$ & 20.0 & $39.2(0.5)$ & $33.9(0.5)$ & $42.6(0.8)$ & $52.9(0.9)$ & 43.7 \\
\hline $52028^{*}$ & 0.793 & & & & $10.3(1.3)$ & 19.8 & $39.9(0.5)$ & $32.9(0.6)$ & $42.1(0.5)$ & $53.3(1.3)$ & 44.3 \\
\hline $52032^{*}$ & 0.803 & & & & $12.8(1.4)$ & 23.2 & $43.5(0.6)$ & $34.4(0.7)$ & $45.3(0.4)$ & $57.6(1.5)$ & 48.1 \\
\hline $52036^{*}$ & 0.814 & & & & $13.8(1.3)$ & 25.0 & $45.0(0.9)$ & $36.6(0.4)$ & $46.4(0.2)$ & $61.9(0.9)$ & 49.7 \\
\hline $52040^{*}$ & 0.825 & & & & $14.7(1.5)$ & 26.2 & $46.0(0.7)$ & $34.6(1.4)$ & $46.7(0.2)$ & $63.3(1.1)$ & 51.0 \\
\hline
\end{tabular}

Notes. For saturated lines the radial velocities (itallic) were obtained by fitting their wings. $1-\sigma$ errors of the mean values are in brackets. ${ }^{(*)}-$ HST STIS spectra.

Z And had two outbursts between JD 45797 and JD 46596, while AG Dra had more than one outburst during the period studied. AX Per, SY Mus and RW Hya are eclipsing systems. Near infrared photometry indicates elipsoidal variations for SY Mus and RW Hya with Roche lobe filling factors of 0.83 and 0.91 respectively and therefore near unity according to Rutkowski et al. (2007). Ineed Mikołajewska (2007) gives reasons for believing that the cool components of most $\mathrm{S}$ type systems fill their Roche lobes.

The radial velocities were measured by Gaussian fits to the whole profile, using the SPLOT programme of IRAF, This averages over any real or instrumental assymmetry. Saturated lines were measured by fitting to the wings. We have checked that the radial velocities determined in this way closely agree with the line centroid radial velocities. It is because of our method that we were able to use emission lines, whose centres are saturated. The nature of our IUE spectra does not enable us to do anything more precise. Let us note however that the HST/STIS spectra of AG Dra suggest some assymmetry of the resonance emission line profiles, with line centres being shifted by apparent absorption on the low wavelength side (Young 2006, private communication as well as the MAST archive).

The errors, indicated in the radial veocity tables, correspond to $1 \sigma$ errors of the respective mean values. They do not indicate systematic errors, such as for instance those of errors in the absolute wavelength scale. However the mean values are based on data of different transitions, so their $\sigma$ values include that due to uncertainty of laboratory wavelengths. The other systematic errors due to the absolute wavelength scale, have been estimated by comparing our radial velocity estimates from different spectra of the same target, taken at the same epoch. In particular we found 14 pairs of IUE spectra ( 4 for Z And, 10 for AG Dra) taken on the same day or one day later. The offset in radial velocity for these pairs is in the range $\Delta v \sim 0-20 \mathrm{~km} \mathrm{~s}^{-1}$, with an offset for 10 pairs of $2-8 \mathrm{~km} \mathrm{~s}^{-1}$, a median value of around $5 \mathrm{~km} \mathrm{~s}^{-1}$ and a mean of $\sim 6.7 \pm 1.3 \mathrm{~km} \mathrm{~s}^{-1}$. There is no difference in the offset between saturated and non-saturated lines, when we compare a spectrum on which a particular line is saturated and another spectrum on which it is not saturated. The corresponding offset for $2 \mathrm{HST} / \mathrm{STIS}$ spectra of AG Dra taken on the same day is only $1.7 \mathrm{~km} \mathrm{~s}^{-1}$.

\section{Results}

Our measured radial velocity means and their $1 \sigma$ errors are tabulated in Table 1 for AX Per, SY Mus and RW Hya, in Table 2 for $\mathrm{Z}$ And and in Table 3 for AG Dra. The phases of the tables are from the ephemeris of Mikołajewska (2003), with respect to inferior conjunction of the giant. The last 8 lines in Table 1 for RW Hya and the last 2 lines in Table 3 for AG Dra are based on HST/STIS spectra. The wavelength of the He II 1640 A line used, is a mean wavelength of the fine structure, given by Clegg et al. (1999). Let us note that this mean is fairly insensitive to the exact physical conditions of line formation; ranges of electron density from $10^{4}$ to $10^{9} \mathrm{~cm}^{-3}$ and temperatures from 10000 to $30000 \mathrm{~K}$ give for case B line formation a shift of $0.007 \AA$ or $1.3 \mathrm{~km} \mathrm{~s}^{-1}$.

Some radial velocities in the tables, including means and the radial velocity of the He II $1640 \AA$ line, plotted against orbital phase, are shown in Fig. 1, for SY Mus, while radial velocity differences with much less scatter are shown in Figs. 2 and 3 for the same symbiotic. Similar figures shown, are 4, 5 and 6 for $Z$ And and 7, 8 and 9 for AG Dra. The difference graphs are with respect to the resonance doublet of C IV, as measurements of the strong lines belonging to this doublet could be made for all the spectra studied by us. Shifts, such as those of the intercombination 
M. Friedjung et al.: Symbiotic line shifts

Table 2. Radial velocities of the UV emission lines (in units of $\mathrm{km} \mathrm{s}^{-1}$ ) for $\mathrm{Z}$ And.

\begin{tabular}{|c|c|c|c|c|c|c|c|c|c|c|c|}
\hline $\begin{array}{l}\text { MJD } \\
\text { IP [eV] }\end{array}$ & $\phi$ & $\begin{array}{c}\text { Si III] } \\
16.3\end{array}$ & $\begin{array}{l}\text { C III] } \\
24.4\end{array}$ & $\begin{array}{l}\mathrm{N} \text { III] } \\
29.6\end{array}$ & $\begin{array}{l}\text { O III] } \\
35.1\end{array}$ & $\begin{array}{c}\text { N IV] } \\
47.4\end{array}$ & $\begin{array}{l}\text { O IV] } \\
54.1\end{array}$ & $\begin{array}{l}\text { Si IV } \\
33.5\end{array}$ & $\begin{array}{l}\text { C IV } \\
47.5\end{array}$ & $\begin{array}{l}\mathrm{N} \mathrm{v} \\
77.5\end{array}$ & $\begin{array}{c}\mathrm{He} \text { II } \\
54.4\end{array}$ \\
\hline 43922 & 0.655 & -6 & -7 & -6 & $-2.6(0.1)$ & -8 & $5.3(1.1)$ & $7.3(2.6)$ & $10.1(1.3)$ & $0.4(2.0)$ & -6 \\
\hline 44075 & 0.856 & -13 & -14 & -14 & $-13.4(1.0)$ & -11 & $-4.4(1.4)$ & 2 & 7.1(1.6) & $-0.2(2.0)$ & -12 \\
\hline 44402 & 0.287 & 0 & -1 & -9 & $-1.8(1.1)$ & -7 & $10.1(2.5)$ & $11.6(2.6)$ & $14.2(0.1)$ & $8.1(1.1)$ & -12 \\
\hline 44480 & 0.391 & -5 & 1 & -4 & $-1.7(4.0)$ & 0.8 & $7.9(1.0)$ & $6.9(6.0)$ & $13.1(0.9)$ & $5.9(0.6)$ & -12 \\
\hline 44719 & 0.706 & 9 & 10 & & 4 & 8 & & & $17.9(0.3)$ & 15 & 1 \\
\hline $45797^{* *}$ & 0.126 & -24 & -18 & -21 & $-27.1(5.1)$ & -29 & $-21.8(0.9)$ & $18.0(4.5)$ & $17.7(1.7)$ & $12.3(4.6)$ & -4 \\
\hline $45863^{* *}$ & 0.213 & -9 & -11 & $-11.8(0.5)$ & $-10.6(2.0)$ & -11 & $-1.9(2.1)$ & $9.0(0.1)$ & $30.7(4.3)$ & $12.5(3.5)$ & 1 \\
\hline 45946 & 0.323 & -8 & -17 & -14 & $-10.0(1.0)$ & -16 & $-1.5(1.5)$ & $7.6(3.8)$ & $16.5(5.4)$ & $2.7(0.8)$ & -14 \\
\hline 45948 & 0.325 & 6 & 10 & 5 & $4.1(0.2)$ & 2 & 11.1(1.6) & $17.6(1.3)$ & $21.7(0.4)$ & $16.3(3.5)$ & -3 \\
\hline 46232 & 0.700 & -6 & -1 & $-0.5(2.8)$ & $-1.9(1.5)$ & -7 & $6.1(1.0)$ & $11.4(2.8)$ & $11.2(0.8)$ & $11.8(1.7)$ & 5 \\
\hline 46233 & 0.701 & 5 & 4 & -4 & $12.1(4.0)$ & 6 & 17 & $23.1(2.7)$ & $24.1(1.3)$ & $22.7(1.6)$ & 1 \\
\hline 46283 & 0.767 & 10 & 3 & 1 & 6 & 14 & $26.2(3.9)$ & & $25.9(3.1)$ & $21.5(0.9)$ & 3 \\
\hline $46595^{* *}$ & 0.178 & -13 & -20 & $-24.6(1.7)$ & $-19.4(1.6)$ & -23 & $-10.9(0.1)$ & $5.4(5.0)$ & $4.4(3.8)$ & $12.1(4.8)$ & -20 \\
\hline $46596^{* *}$ & 0.179 & -7 & -15 & $-14.7(1.4)$ & $-11.4(1.2)$ & -11 & $-3.5(1.6)$ & $10.7(1.3)$ & $18.5(2.4)$ & & -11 \\
\hline 46715 & 0.336 & -21 & -27 & $-19.4(1.9)$ & $-19.4(1.9)$ & -28 & $-12.8(1.3)$ & $-4.3(1.7)$ & $-2.3(1.4)$ & $-2.5(0.1)$ & -21 \\
\hline 46778 & 0.419 & -8 & -16 & $-6.3(2.1)$ & $-6.2(0.1)$ & -8 & $-1.1=(1.1)$ & $5.0(0.8)$ & $8.1(0.4)$ & $3.7(2.8)$ & -20 \\
\hline 46962 & 0.662 & 7 & 11 & 11 & $10.4(1.7)$ & 7 & $16.8(4.3)$ & $22.5(0.5)$ & $24.0(0.7)$ & $19.2(2.3)$ & -8 \\
\hline 47081 & 0.819 & 0 & 0 & -2 & $-1.0(3.1)$ & -5 & 10 & & $19.0(1.8)$ & $16.3(4.7)$ & 6 \\
\hline 47101 & 0.845 & -10 & -16 & -13 & $-8.3(1.5)$ & -13 & $-0.1(3.1)$ & 8 & 11.1(1.0) & $9.4(5.3)$ & -3 \\
\hline 47195 & 0.969 & -23 & -18 & & -15 & & & & $4.4(0.1)$ & & -16 \\
\hline 47327 & 0.143 & -33 & -37 & -34 & -31 & -39 & $-30.3(2.1)$ & $-13.5(1.2)$ & $-6.6(0.8)$ & -13 & -33 \\
\hline 47713 & 0.650 & -2 & -2 & -11 & $-3.3(2.5)$ & -5 & $2.5(1.2)$ & $4.6(3.5)$ & $11.4(1.2)$ & $7.4(1.1)$ & -8 \\
\hline 47845 & 0.825 & -8 & -8 & -8 & $-6.8(1.90)$ & -12 & $-2.7(0.8)$ & $7.1(0.1)$ & $11.1(2.6)$ & $6.4(1.3)$ & -7 \\
\hline 47885 & 0.878 & -16 & -18 & -15 & -26 & -21 & $-17(3.7)$ & & $-1.5(1.5)$ & -3 & -20 \\
\hline 48092 & 0.150 & -1 & 3 & & -4 & -8 & 2 & & $25.1(0.5)$ & & -4 \\
\hline 48929 & 0.254 & -9 & -8 & -7 & -7 & & 0 & & $10.8(4.6)$ & 10.9 & -20 \\
\hline 48997 & 0.343 & -7 & 7 & -4 & $-3.9(0.3)$ & -1 & $10.5(0.5)$ & $9.4(0.9)$ & $12.2(1.0)$ & $7.6(3.0)$ & -9 \\
\hline 49163 & 0.563 & 6 & 8 & 0 & $3.7(2.3)$ & 0 & $8.4(0.1)$ & $10.3(2.8)$ & $15.4(0.5)$ & $9.8(0.4)$ & -8 \\
\hline 49229 & 0.650 & 7 & 10 & 5 & $11.0(0.4)$ & 9 & $23.1(1.0)$ & $20.5(0.5)$ & $20.4(0.3)$ & $18.8(1.2)$ & 5 \\
\hline 49617 & 0.161 & -30 & -18 & & -21 & -27 & -15 & & $2.2(1.1)$ & & -24 \\
\hline 49678 & 0.241 & -7 & -7 & & $-14.9(0.1)$ & -18 & $-10.3(2.3)$ & -4 & $7.0(0.2)$ & 6.8 & -18 \\
\hline 49728 & 0.307 & -6 & 0 & & $-9.3(1.5)$ & -2 & $-0.2(1.9)$ & $4.5(0.2)$ & $7.8(0.5)$ & $1.0(4.0)$ & -17 \\
\hline 49898 & 0.523 & -21 & -14 & -20 & $-18.3(1.3)$ & -20 & -9 & $-8.3(4.0)$ & $0.2(1.7)$ & $-8.8(1.1)$ & -30 \\
\hline 49963 & 0.617 & -3 & 3 & -15 & $-5.1(0.6)$ & -13 & & 6 & $7.9(1.0)$ & 2 & -12 \\
\hline 50104 & 0.802 & -16 & -7 & & $-13.4(2.6)$ & -11 & & & $4.6(0.2)$ & & -16 \\
\hline 50104 & 0.802 & -21 & -25 & -19 & $-17.0(0.6)$ & -18 & $-11.3(4.2)$ & $-3.0(0.2)$ & $-1.4(1.0)$ & $3.0(0.6)$ & -18 \\
\hline
\end{tabular}

Notes. For saturated lines the radial velocities (itallic) were obtained by fitting their wings. ${ }^{(*)} 1-\sigma$ errors of the mean values are in brackets. ${ }^{(* *)}$ - spectra taken during outbursts. The profiles on spectra taken during the outburst on MJD 46353-46462 are very complex and they cannot be characterised by single radial velocity.

lines relative to C IV, depend on the shift of C IV itself. The values for Si IV are not plotted, but their behaviour can be seen from the tables.

In the graphs mentioned, the open circles are of fits of the line centres to a Gaussian for IUE observations in quiescence, while the outburst IUE observation fits of the line centres for $\mathrm{Z}$ And and AG Dra are plotted as filled circles. Open and filled triangles denote corresponding fits of the line wings in quiescence and outburst, when the centres are saturated. Finally an X is used to plot HST/STIS data of AG Dra. The dotted curve in these figures is the radial velocity curve of the cool component from Belczyński et al. (2000), taking phase 0 as that of inferior conjuction of that component.

We mainly show and study the difference graphs and values, which are much less affected by the systematic errors, as shown above. In the case of SY Mus, Fig. 2 shows that the $C$ IV radial velocity is redshifted and almost constant, while He II tends to follow the hot compoment with a blueshift. One can see from Fig. 2 that the mean $\mathrm{C}$ IV and $\mathrm{N}$ V redshifts are almost the same without systematic variations of the difference between them with orbital phase, while in addition the Table 1 C IV - Si IV differences are small and slightly positive. The Fig. 2 C IV - He II $1640 \AA$ radial velocity graph shows a relative redshift of C IV with a minimum perhaps near phase 0.5 , this being a reflection of the radial velocity variation of He II by itself. The redshift of the C IV resonance with respect to the intercombination lines of SY Mus varies fairly smoothly with orbital phase, with a maximum approaching $30 \mathrm{~km} \mathrm{~s}^{-1}$ somewhat before phase 0 and a much smaller minimum (Fig. 3).

There are far more measurements for $\mathrm{Z}$ And which shows comparable effects. The O IV] and He II $1640 \AA$ radial velocities show a maximum which seems to be in phase with the radial velocity of the hot component at least during quiescence (Fig. 4). The He II line may have a small tendency to have a blueshift. The difference graphs of $\mathrm{Z}$ And give clear results. Shifts of N V relative to $\mathrm{C}$ IV in Fig. 5 are at least during quiescence almost always small and independent from orbital phase. We may note that the C IV - Si IV differences given by the table have a certain amount of scatter, but are usually small (less than $10 \mathrm{~km} \mathrm{~s}^{-1}$ and positive). Figure 5 shows that the $\mathrm{C}$ IV - He II radial velocity is in phase with the cool component, or that the He II radial velocity relative to that of CIV is in phase with the hot 
A\&A 512, A80 (2010)

Table 3. Radial velocities of the UV emission lines (in units of $\mathrm{km} \mathrm{s}^{-1}$ ) for AG Dra.

\begin{tabular}{|c|c|c|c|c|c|c|c|c|c|c|}
\hline MJD & $\phi$ & $\mathrm{Si}$ III] & C III] & O III] & N IV] & O IV] & Si IV & CIV & $\mathrm{NV}$ & He II \\
\hline $\mathrm{IP}[\mathrm{eV}]$ & & 16.3 & 24.4 & 35.1 & 47.4 & 54.1 & 33.5 & 47.5 & 77.5 & 54.4 \\
\hline 44418 & 0.421 & -138 & & $-141.8(0.9)$ & -137 & $-133.9(2.0)$ & -132 & $-131.9(0.6)$ & $-145.3(5.0)$ & -149 \\
\hline 44418 & 0.421 & -142 & -137 & $-143.1(1.3)$ & -142 & $-137.0(1.5)$ & -142 & $-138.2(1.0)$ & $-145.0(2.9)$ & -155 \\
\hline $44698^{* *}$ & 0.932 & & -145 & $-156.3(4.8)$ & -159 & $-151.4(0.7)$ & $-156.7(1.8)$ & $-149.9(0.1)$ & $-149.6(1.7)$ & -162 \\
\hline $44700^{* *}$ & 0.934 & -148 & -154 & $-153.0(1.9)$ & -154 & $-143.8(1.2)$ & $-144.6(0.6)$ & $-144.3(0.1)$ & $-147.9(3.0)$ & -152 \\
\hline $44719^{* *}$ & 0.969 & -141 & -145 & -153 & -156 & $-146.1(4.6)$ & $-148.7(0.4)$ & $-147.5(1.1)$ & $-146.3(0.6)$ & -158 \\
\hline $44719^{* *}$ & 0.969 & -146 & -144 & $-153.5(1.4 .0)$ & -155 & $-144.2(0.8)$ & $-145.4(1.2)$ & $-144.7(1.4)$ & $-142.9(0.1)$ & -159 \\
\hline $44820^{* *}$ & 0.154 & -157 & -145 & $-156.5(1.3)$ & -161 & $-149.6(1.6)$ & $-154.7(2.3)$ & $-152.5(0.2)$ & $-158.0(2.6)$ & -168 \\
\hline $44944^{* *}$ & 0.379 & -160 & -179 & $-166.3(0.3)$ & -168 & $-162.1(0.6)$ & $-163.3(1.5)$ & $-170.6(0.1)$ & $-169.7(1.0)$ & -185 \\
\hline $44950^{* *}$ & 0.390 & -153 & -161 & $-160.4(1.7)$ & -162 & $-158.3(3.0)$ & $-159.3(1.8)$ & $-160.6(1.5)$ & $-168.3(3.9)$ & -188 \\
\hline $44950^{* *}$ & 0.390 & -154 & & -157 & -166 & -160 & $-150.7(2.7)$ & $-159.7(1.0)$ & $-160.0(4.6)$ & -179 \\
\hline 45492 & 0.378 & -143 & -140 & $-146.6(0.5)$ & -145 & $-137.1(1.7)$ & $-145.8(0.1)$ & $-141.7(0.7)$ & -145 & -158 \\
\hline 46138 & 0.554 & -144 & & $-151.3(0.7)$ & -152 & $-142.2(3.2)$ & $-149.9(0.8)$ & $-148.0(0.7)$ & -153 & -165 \\
\hline 46155 & 0.585 & -153 & & & -148 & -148 & & $-150.7(1.4)$ & $-153.1(3.8)$ & -168 \\
\hline 46374 & 0.984 & & -152 & -157 & -154 & -136 & & $-137.0(0.3)$ & -139 & -149 \\
\hline 47827 & 0.631 & -156 & & $-160.7(0.8)$ & -163 & $-148.9(5.9)$ & -152 & $-150.5(0.8)$ & -150 & -164 \\
\hline 47828 & 0.632 & -152 & & & & & & $-148.1(7.7)$ & -157 & -159 \\
\hline 48225 & 0.355 & -134 & & $-145.7(1.3)$ & -140 & -140 & & $-135.9(0.4)$ & $-132.1(3.1)$ & -151 \\
\hline 48812 & 0.424 & & & & -149 & -137 & & $-149.6(7.1)$ & -157 & -157 \\
\hline 48878 & 0.545 & & & & & & & $-137.3(2.9)$ & -128 & -140 \\
\hline 48898 & 0.581 & -124 & & & & & & $-125.4(3.4)$ & -126 & -139 \\
\hline 48960 & 0.695 & -142 & & -146.2 & -148 & $-138.2(1.4)$ & -143 & $-139.4(2.2)$ & $-138.9(1.0)$ & -154 \\
\hline 49058 & 0.873 & -161 & & $-165.7(3.6)$ & -163 & $-152.2(4.4)$ & -157 & $-150.9(2.0)$ & -149 & -166 \\
\hline $49533^{* *}$ & 0.737 & -140 & -134 & -145 & -144 & $-140.8(2.1)$ & $-146.7(4.6)$ & $-142.7(0.6)$ & $139.2(0.7)$ & -143 \\
\hline $49533^{* *}$ & 0.737 & & & -154 & -153 & -142 & -148 & $-148.0(1.3)$ & -150 & -153 \\
\hline $49538^{* *}$ & 0.748 & -133 & -143 & -150 & -153 & $-138.2(2.9)$ & $-142.9(1.6)$ & $-144.9(0.1)$ & $-134.9(1.3)$ & -152 \\
\hline $49540^{* *}$ & 0.750 & -164 & & $-172.4(0.2)$ & -167 & $-153.4(5.3)$ & $-163.3(1.1)$ & $-164.3(1.6)$ & $-154.6(2.9)$ & -173 \\
\hline $49543^{* *}$ & 0.755 & & & -170 & -144 & -125 & -143 & $-136.5(0.8)$ & $-110.5(1.8)$ & -120 \\
\hline $49543^{* *}$ & 0.755 & -151 & & -167 & -148 & -140 & $-133.7(1.6)$ & $-143.0(0.7)$ & -130 & -130 \\
\hline $49546^{* *}$ & 0.762 & & & -168 & & & & $-164.6(6.0)$ & -143 & -165 \\
\hline $49561^{* *}$ & 0.789 & -144 & & -143 & & & & $-147.2(3.5)$ & -132 & -158 \\
\hline $49562^{* *}$ & 0.791 & -151 & -146 & -164 & -160 & -154 & $-153.4(4.5)$ & $-154.8(0.9)$ & $-148.0(5.6)$ & -169 \\
\hline $49613^{* *}$ & 0.884 & -132 & -146 & $-145.6(4.7)$ & -145 & $-145.3(3.9)$ & $-146.6(5.3)$ & $-137.9(0.1)$ & -138 & -154 \\
\hline $49693^{* *}$ & 0.029 & -140 & & $-141.9(0.4)$ & -143 & $-134.9(0.5)$ & -130 & $-134.6(1.0)$ & $-133.5(0.8)$ & -154 \\
\hline $49693^{* *}$ & 0.029 & -147 & & $-149.0(1.5)$ & -149 & $-141(3.1)$ & $-144.7(0.2)$ & $-140.8(0.6)$ & $-140.9(2.1)$ & -157 \\
\hline $49846^{* *}$ & 0.307 & -153 & & -154 & -154 & $-144.0(0.5)$ & & $-145.5(1.2)$ & -151 & -162 \\
\hline $49846^{* *}$ & 0.307 & -154 & -142 & $-158.1(2.0)$ & -154 & $-147.7(0.9)$ & $-151.8(0.7)$ & $-152.5(0.9)$ & -154 & -169 \\
\hline $49927^{* *}$ & 0.456 & -157 & -147 & $-162.6(1.4)$ & -161 & $-150.5(1.8)$ & $-151.9(0.4)$ & $-148.2(1.2)$ & $-153.0(1.2)$ & -155 \\
\hline $49928^{* *}$ & 0.456 & -146 & -138 & -154 & -156 & -143 & -143 & $-141.0(0.3)$ & -145 & -161 \\
\hline $49928^{* *}$ & 0.456 & -152 & & -161 & & & & $-138.5(1.1)$ & & -161 \\
\hline $49975^{* *}$ & 0.541 & -154 & -137 & $-160.5(0.4)$ & -160 & $-153.0(1.8)$ & $-154.9(3.4)$ & $-150.9(0.5)$ & $-152.8(2.6)$ & -159 \\
\hline $50016^{* *}$ & 0.618 & -151 & -150 & $-157.6(1.5)$ & -160 & $-151.8(1.4)$ & $-151.1(1.3)$ & $-150.3(0.7)$ & -152 & -170 \\
\hline $50064^{* *}$ & 0.705 & -140 & -128 & -143.1 & -143 & $-134.7(0.3)$ & $-134.2(4.5)$ & $-131.4(0.9)$ & $-138.4(1.2)$ & -155 \\
\hline $50128^{* *}$ & 0.823 & -147 & & -151 & -145 & -135 & -136 & $-130.8(0.9)$ & -133 & -147 \\
\hline $52756^{*}$ & 0.608 & & & $-148.2(0.1)$ & -148.4 & $-138.9(0.6)$ & $-141.8(1.0)$ & $-138.4(1.5)$ & $-134.9(0.6)$ & -151 \\
\hline $52756^{*}$ & 0.608 & & & $-150.0(0.1)$ & -150.0 & $-140.1(0.9)$ & $-143.4(0.6)$ & $-140.1(1.6)$ & $-136.1(0.6)$ & -152 \\
\hline
\end{tabular}

Notes. For saturated lines the radial velocities (itallic) were obtained by fitting their wings. $1-\sigma$ errors of the mean values are in brackets. ${ }^{(*)}-$ HST STIS spectrum. ${ }^{(* *)}$ - spectra taken during outbursts.

component. The situation is somewhat less certain for O IV], which may reach a maximum radial velocity relative to that of C IV earlier. C IV is however redshifted with respect to O IV] at most orbital phases. Figure 6 shows radial velocity differences between C IV and other intercombination lines, which have a similar orbital phase dependence, but which are generally more positive than the O IV] difference. Let us note that the phase dependence would appear to be somewhat different than that of SY Mus. In connection with these results let us note that IUE wavelengths of O IV] are usually less accurate than those of He II.

AG Dra shows fewer clear effects. Unlike in quiescence there are enough He II and O IV] measurements during outburst, to indicate that they appear to follow the hot component at least at such times (Fig. 7). The difference graphs of Fig. 8 show constant differences at almost all times, C IV having a small redshift relative to He II. This constancy was violated during outburst between JD 49538 and 49613 at phases of $0.7-0.9$ (Table 1). Figure 9 shows the radial velocity shift between the C IV and intercombimation lines excluding O IV]; except perhaps for C III] in outburst, no clear sign of any orbital variation is seen, while there might be a significant redshift for line centre meaurements of $\mathrm{C}$ IV relative to $\mathrm{C}$ III] in quiescence. As can be seen in Table 3 the higher quality HST/STIS spectra show a clear redshift of O IV] relative to the other intercombination lines, produced by ions with a lower ionization potential.

Figures are not shown for the other symbiotics for which the observations have poor phase coverage. However most RW Hya measurements, based on HST/STIS spectra are very accurate. 
M. Friedjung et al.: Symbiotic line shifts
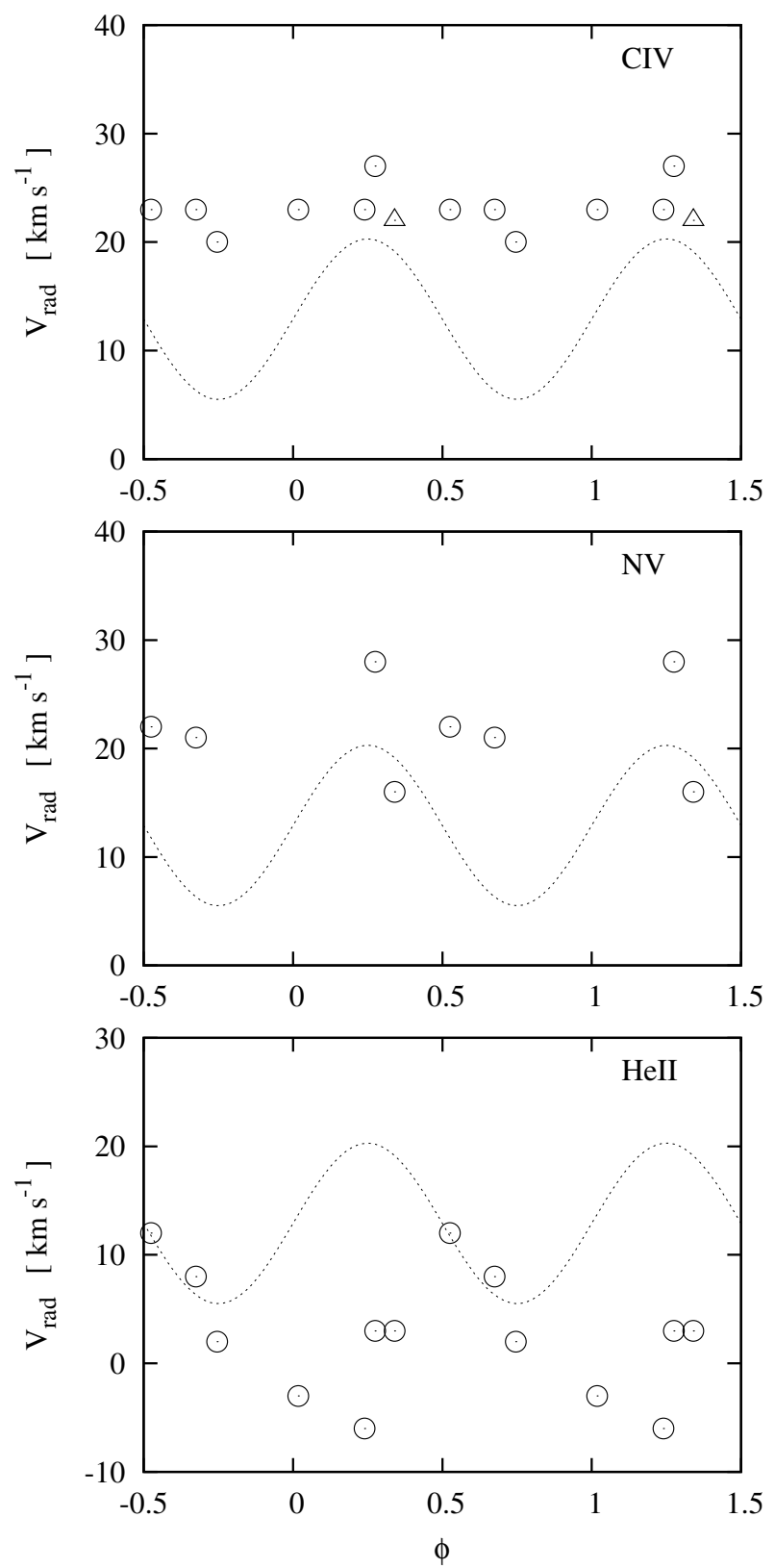

Fig. 1. Radial velocities for SY Mus. The dashed line shows the orbital velocity of the cool giant. The symbols are explained in the text.

The C IV - Si IV radial velocity differences are small and positive, with very little variation between 8.7 and $12.1 \mathrm{~km} \mathrm{~s}^{-1}$. The $\mathrm{C}$ IV - N V difference shows however a more complex behaviour. The first 2 values, based on IUE spectra are similarly small (7 and $4 \mathrm{~km} \mathrm{~s}^{-1}$ ), while the later values from HST/STIS spectra as well as being closely spaced in time at phases not very far from that of the second IUE spectrum, but taken two decades later, are curiously negative, decreasing between the phases 0.749 and 0.825 from -6.5 to $-16.6 \mathrm{~km} \mathrm{~s}^{-1}$. In this way $\mathrm{N} \mathrm{V}$ appears to be more redshifted with respect to the intercombination lines than $\mathrm{C}$ IV. The C IV redshift relative to the intercombination lines, deduced from the HST/STIS spectra, decreases with the ionization potential of the corresponding ions, being much less for O IV] than for O III] and N IV]. The He II $1640 \AA$ line appears to be for all but the first observation strongly redshifted with respect to the systemic radial velocity of 12.4 or $12.9 \mathrm{~km} \mathrm{~s}^{-1}$, but its radial velocity may be compatible with that of the compact component


Fig. 2. N v and He II radial velocity differences for SY Mus. The dashed line shows the orbital velocity of the cool giant. The symbols are explained in the text.

at the phases of the observations, if this component is much less massive than the cool one. However the radial velocity variations of He II and the intercombination lines, deduced from the HST/STIS spectra, would appear to be in phase with the orbital radial velocity variations of the cool one.

The AX Per spectra are all for phases very close to conjunction. The C IV, N V and Si IV lines have similar radial velocities, which are redshifted with respect to the intercombination lines, including O IV.

A few line profiles of Z And and RW Hya, obtained from the spectra, are shown in Figs. 10 and 11. They will be considered in the discussion of our results in order to better understand the reasons for the radial velocity shifts.

\section{Discussion}

Let it first be emphasized, that the differences between the C IV mean resonance line radial velocity and the means of other resonance doublets, are generally much smaller than the difference between the CIV resonance line mean and the intercombonation line means for SY Mus, Z And and AX Per in many orbital phases. This clearly indicates that any explanation of the relative shift between the resonance and the intercombination line radial velocities, which is not the same for the different resonance line doublets, cannot work. The shift is in many orbital phases much larger than the $1 \sigma$ errors given in the tables, which shows in addition that shifts between either the different resonance or the intercombination lines of the same ion are small.

We can immediately draw the conclusion that it may be difficult to explain the relative radial velocity shift between the 

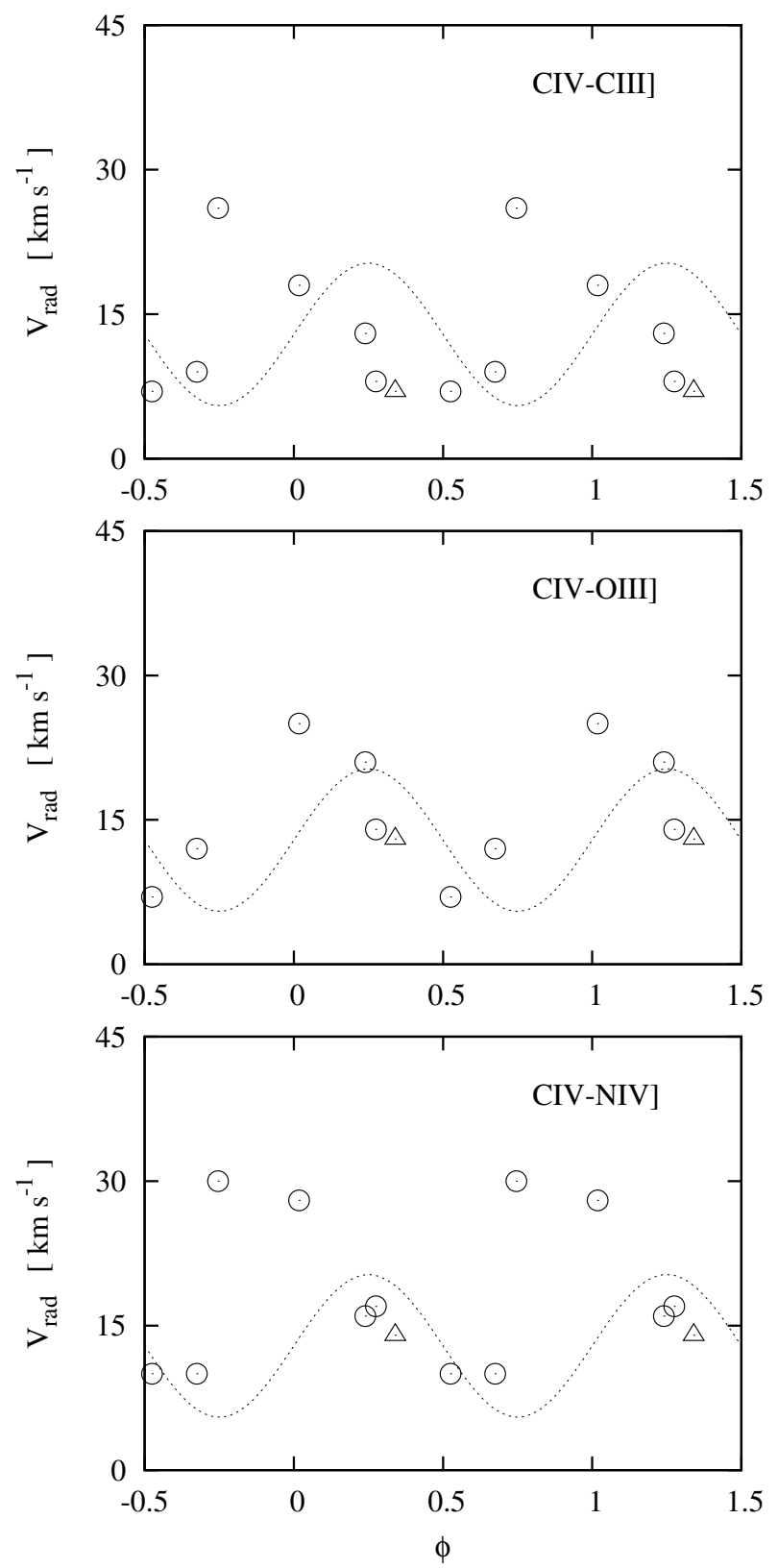

Fig. 3. Intercombination line radial velocity differences for SY Mus. The dashed line shows the orbital velocity of the cool giant. The symbols are explained in the text.

optically thick resonance lines and the intercombination lines by a resonance line radiative transfer effect, because the different optical thicknesses of the resonance line might be expected at first sight to produce different shifts.

Line radial velocities can be influenced by various effects. We need to first examine such effects, before discussing what is observed. Among the former we shall in the first three subsections consider the following.

\subsection{Effects due to the presence of interstellar lines on shifted resonance line}

Interstellar absorption components can be superposed on the resonance emission lines and can distort the line profiles. Such an effect should not be present for the symbiotic systems with large systemic velocities $\left(-116.5\right.$ and $-148.3 \mathrm{~km} \mathrm{~s}^{-1}$ of AX Per and
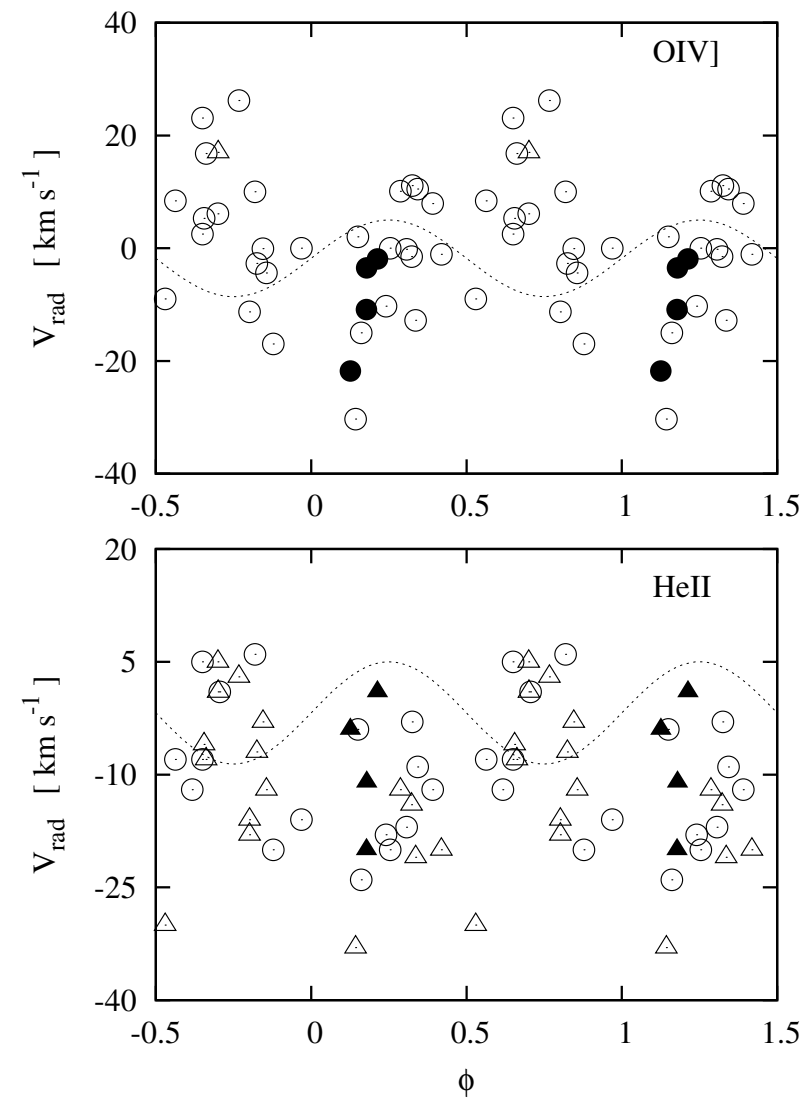

Fig. 4. Intercombination O IV] and He II radial velocities for Z And. The dashed line gives the orbital velocity of the cool giant. The symbols are expained in the text.

AG Dra respectively). In the case of the others we can look for narrow at least partly interstellar absorption lines in stars, which are close in the sky, but which do not have smaller linear distances from the observer. Let us note that this test for the effect of interstellar absorption is not completely watertight. Radiation from the hot component of a symbiotic binary could ionize the nearby interstellar medium, so producing interstellar absorption from highly ionized atoms, without such lines being seen in the spectra of other stars in similar lines of sight.

The estimated distance of SY Mus is $850 \mathrm{pc}$ according to Schmutz et al. (1994), while TU Mus, a $\beta$ Lyr type eclipsing variable with a $\mathrm{O}$ star component, has a distance of $2.1 \mathrm{kpc}$ according to Penny et al. (2008) and is only $0.3^{\circ}$ in the sky from SY Mus. The stronger N V $1238 \AA$ doublet resonance line absorption of TU Mus is weak as is also the stronger Si IV $1393 \AA$ doublet resonance line. The shifts of $\mathrm{NV}$ and $\mathrm{Si}$ IV relative to C IV of SY Mus are both near zero however, suggesting hardly any effect of interstellar absorption in the direction of TU Mus and SY Mus.

High resolution HST/STIS spectra are available for RW Hya and we can directly inspect the spectra for the presence of narrow absorption lines. In fact extremely narrow absorption lines are seen, superposed on N V $1242 \AA$ emission as well as in other places, though they may rather be circumstellar (see discussion below on the iron forest).

$\mathrm{Z}$ And has a distance of $1.5 \mathrm{kpc}$ according to Mikołajewska and Kenyon (1996), while HD218195, separated $10.3^{\circ}$ in the sky, has a distance of $2.9 \mathrm{kpc}$ according to Patriarchi et al. (2001). There is neither any sign of $\mathrm{N} \mathrm{V}$ absorption nor of that of $\mathrm{C}$ IV within the noise. In any case the relative shifts of $\mathrm{N} \mathrm{V}, \mathrm{C}$ IV 



Fig. 5. Intercombination O IV] as well as $\mathrm{N} \mathrm{V}$ and He II radial velocity differences for $\mathrm{Z}$ And. The dashed line shows the orbital velocity of the cool giant. The symbols are explained in the text.

and Si IV in the Z And spectra, which might be expected not to be affected in the same way by interstellar absorption, are usually small at many orbital phases compared with the shift between $\mathrm{C}$ IV and the intercombination lines.

\subsection{Effects due to the absorption component of a P Cygni profile of shifted resonance lines}

It should first be noted that any explanation involving a P Cygni absorption component of a line at a shorter wavelength than the line emission studied has a difficulty when the continuum is weak. In that case, line absorption can however still be produced by an overlying layer with the same radial velocity as regions from which part of the line emission comes. It is this kind of explanation, which we shall use to understand many observations of the resonance lines. In this connection, we can mention
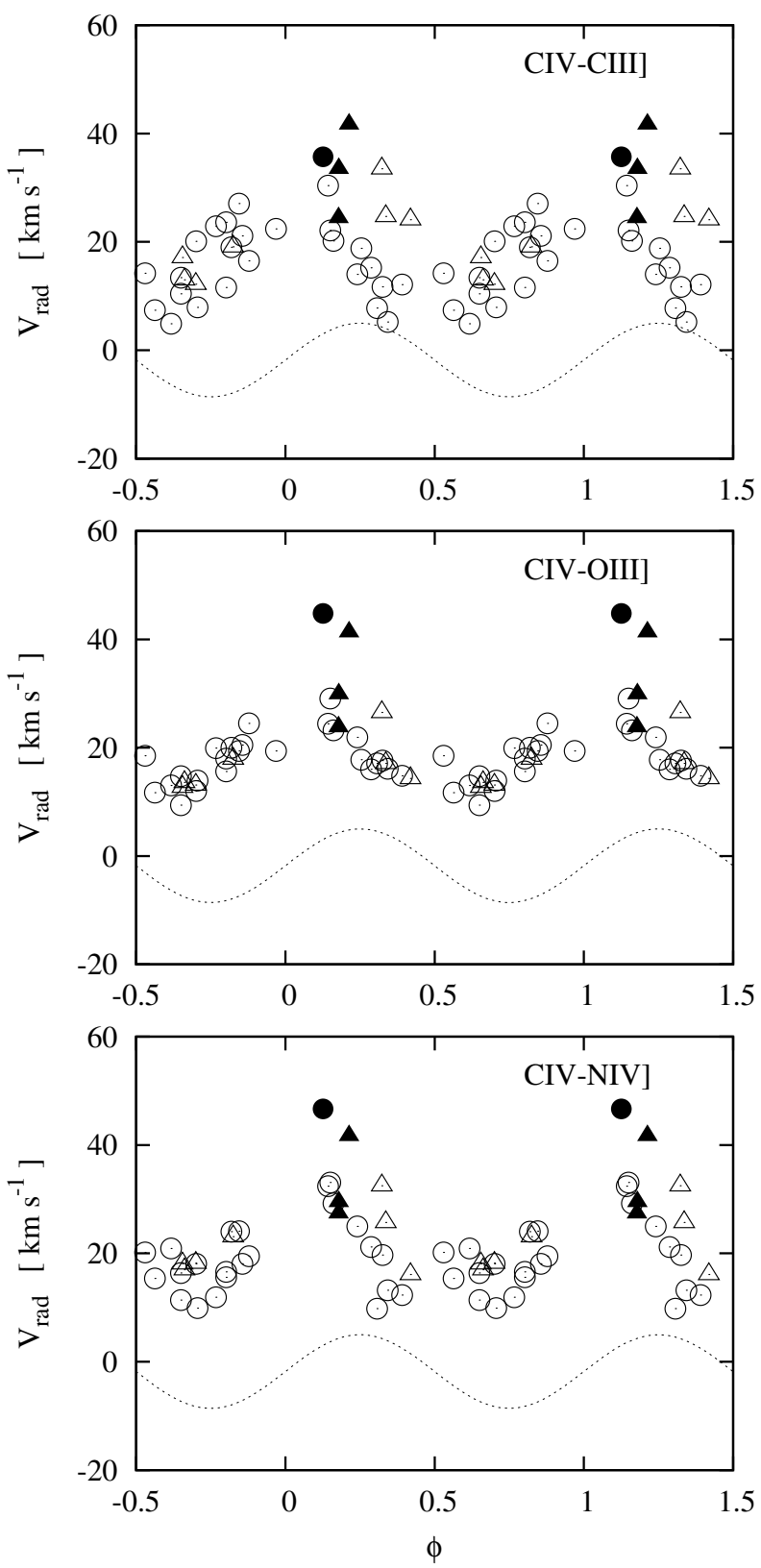

Fig. 6. Intercombination line radial velocity differences of less ionized atoms for $\mathrm{Z}$ And. The dashed line shows the orbital velocity of the cool giant. The symbols are explained in the text.

that Young et al. (2005) suggest that the O VI P Cygni profile of AG Dra is affected by absorption of a false continuum, that is by a continuum enhanced by the presence of electron scattering wings of the line. However the $1 / \mathrm{e}$ width of electron scattering wings is $550 \mathrm{~km} \mathrm{~s}^{-1}$ at $10000 \mathrm{~K}$ for one scattering. If much narrower line emission is only seen, with no evidence of wings with at least this width, such an explanation will not work for any geometry of an electron scattering region relative to that of line formation. In particular in the case of CI Cyg, previously studied by Mikołajewska et al. (2006), there is no evidence of broad wings for the $\mathrm{C}$ IV resonance lines, extending to much more than $100 \mathrm{~km} \mathrm{~s}^{-1}$ in the HST/GHRS spectrum (see Fig. 1 of that paper). 

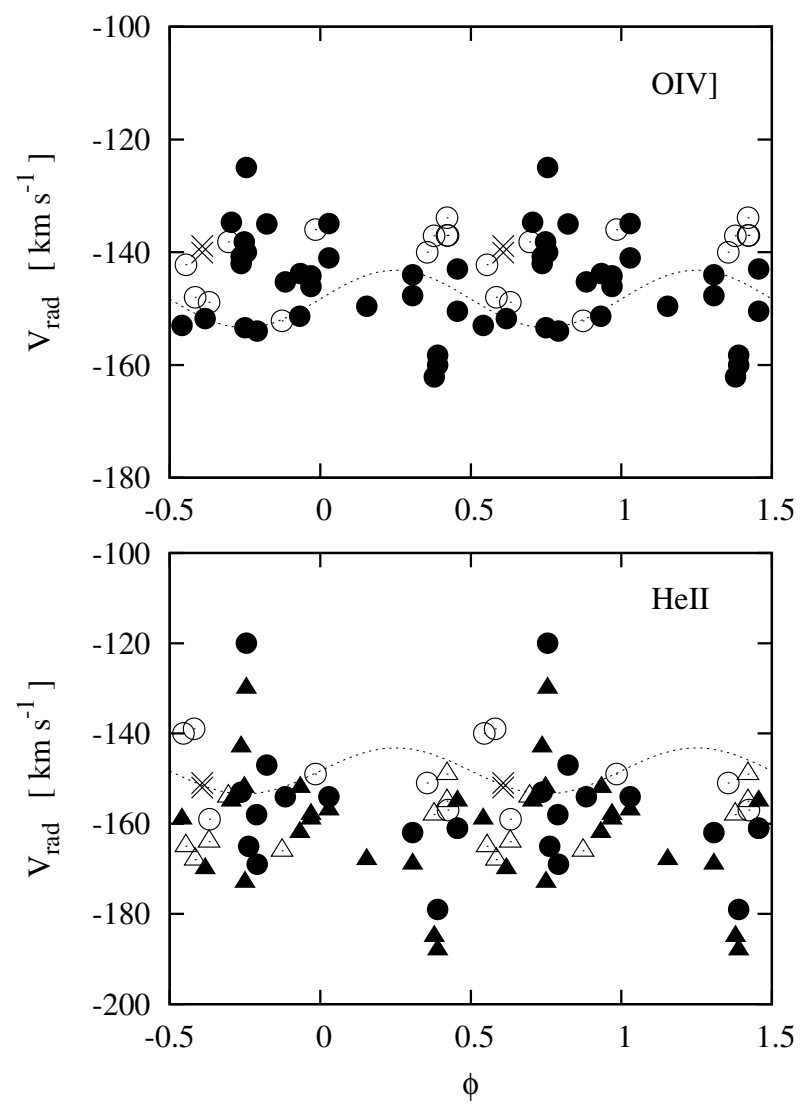

Fig. 7. OIV] intercombination line and He II radial velocities for AG Dra. The dashed line shows the orbital velocity of the cool giant. The symbols are explained in the text

\subsection{Analysis of iron forest absorption and plausible line shifts of lines producing pumping by accidental resonance (PAR)}

The importance of iron forest absorption by the low velocity wind of the cool component, leading to pumping by accidental resonance (PAR), needs to be checked. The effect of the iron forest is well known since the work of Shore and Aufdenberg (1993). In principle such an effect could be quite large, though in fact we shall find usually no large influence of this kind of effect on the radial velocities of the lines studied by us. A major problem of the Shore and Aufdenberg calculations is moreover that the oscillator strengths, used for the lines, are not always reliable. This is for instance the case for the $4 s-4 p^{*}$ transitions involving high parent terms (sometimes refered to as $4 \mathrm{~s}^{*}$ and $4 \mathrm{p}^{*}$ transitions) superposed on short wavelength region IUE emission lines. We can in such a way explain why for instance lines excited or "pumped" as a result of absorption of emission by the C IV $1548 \AA$ line are generally observed unlike those from the C IV $1550 \AA$ line (Erikson et al. 2006).

We can, in order to test for effects of the iron forest, firstly look for the presence of emission lines, emitted in the wind of the cool component from levels "pumped" as a result of absorption of the emission of lines, studied in this paper. The amount of pumping clearly depends on the widths of the lines which can pump. Pumping is summarized for different symbiotic binaries by Eriksson et al. (2006).

In the case of Z And, C IV $1548 \AA$ pumps two channels, while Si IV $1393 \AA$ probably pumps another channel. The $3 d^{7} a^{4} F_{9 / 2}-$ $3 d^{6}\left({ }^{3} \mathrm{G}\right) 4 \mathrm{p} \mathrm{y}{ }^{4} \mathrm{H}_{11 / 2}$ channel pumps 10 observed Fe II emission


Fig. 8. Intercombination line O IV], N v, He II radial velocity differences with the orbital radial velocity of the cool giant for AG Dra. The symbols are explained in the text.

lines at 2772, 2493, 2481, 2459, 2436, 2228, 2220, 2211, 2168 and $1975 \AA$. The $3 \mathrm{~d}^{7} \mathrm{a}^{4} \mathrm{P}_{1 / 2}-4 \mathrm{p} \mathrm{w} \mathrm{w}_{3 / 2}$ channel, pumped by the same C IV line produces the observed 2979.95, 2483, 2479.98 and $1965 \AA$ lines. The 3 Fe II lines probably pumped by Si IV through the $3 d^{6}\left({ }^{5} \mathrm{D}\right) 4 \mathrm{~s} \mathrm{a}{ }^{6} \mathrm{D}_{7 / 2}-3 \mathrm{~d}^{6}\left({ }^{1} \mathrm{G}\right) 4 \mathrm{p} \mathrm{x}^{2} \mathrm{H}_{9 / 2}$ channel are at 2588, 25492 and $1793 \AA$. Correcting the fluxes for the radiation absorbed from the pumping lines, before being re-emitted in the pumped lines, gives nearly "optically thin" flux ratios near 

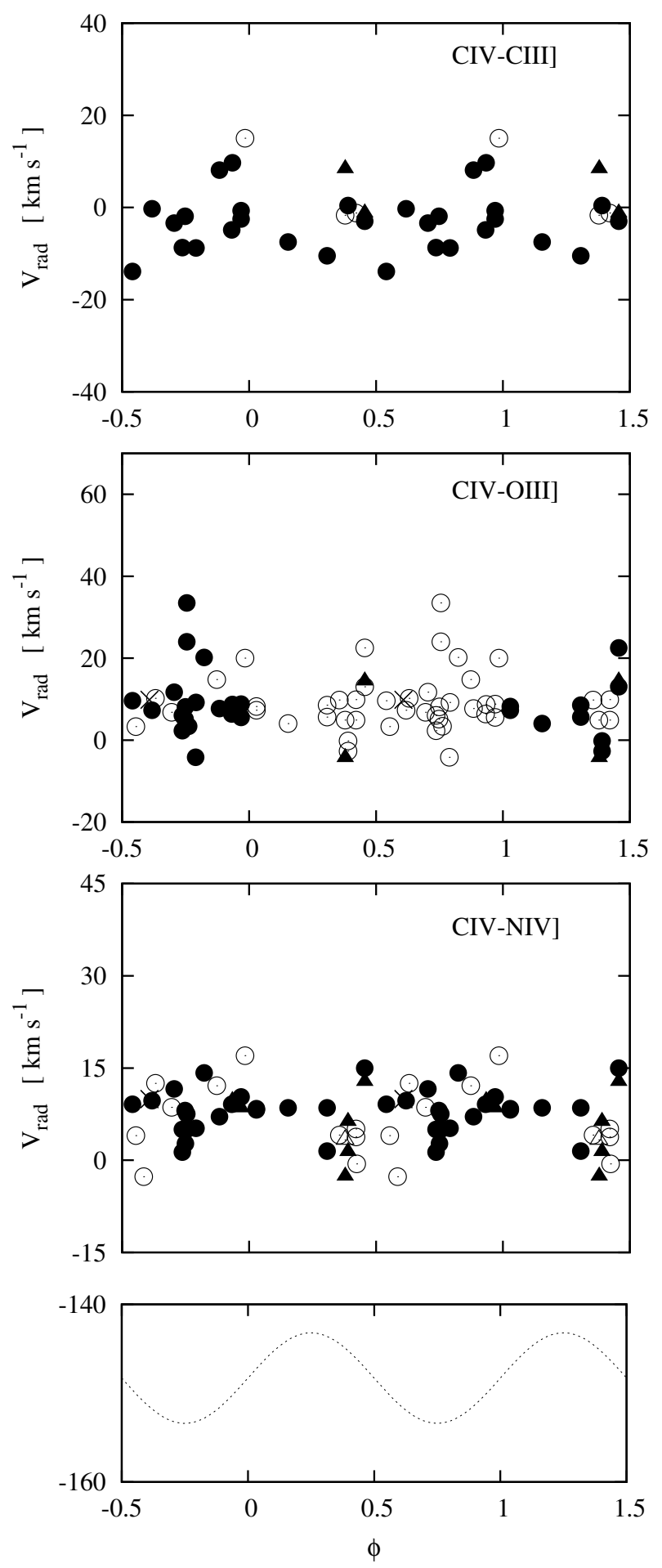

Fig. 9. Intercombination line radial velocity differences of less ionized atoms with the orbital radial velocity of the cool giant for AG Dra. The symbols are explained in the text.

2 for the C IV and Si IV doublets, except in the case of the C IV doublet near phase zero in outburst, when probably blueshifted P Cygni absorption (see Fig. 10) is visible. In addition pumping by the O III] intercombination line at $1660 \AA$ may be responsible for weak emission features in the spectrum of $\mathrm{Z}$ And at 2784.5 and $2728.2 \AA$. However we do not see any systematic velocity shift of the $\mathrm{O}$ III] line with respect to the other intercombination lines.
Pumping by the first $1548 \AA \mathrm{C}$ IV line channel produced 4 observed Fe II emission lines in the spectrum of SY Mus. The $\mathrm{C}$ IV flux ratio, correcting for pumping again gives an "optically thin" value of 2 .

Obtaining a clear result is more difficult for AG Dra. Emission lines, which could be produced by pumping, are also observed sometimes in absorption. However in a private communication P.R. Young states that he sees lines pumped by C IV, He II (1640 ̊ and $1085 \AA$ ), N IV] $1486 \AA$ as well as by O III] $1660 \AA$. We have however seen no sign of Fe II lines pumped by two possible He II $1640 \AA$ channels for AG Dra so any effect of pumping on the He II profile is small. In any case, let us note that pumping may be less important for this metal underabundant symbiotic system.

The C IV $1548 \AA$ line pumps the Fe II $y^{4} H_{11 / 2}$ channel in the spectrum of RW Hya, but lines pumped by this mechanism are not observed in the spectrum of AX Per, suggesting no strong iron forest absorption for the latter symbiotic. We must in the former RW Hya case note in addition, that the HST/STIS spectra show strong narrow absorption components, superposed especially on the N V $1242 \AA$ profile and on the last date on the Si IV profile.

In any case we can note, that the already mentioned small $1 \sigma$ values corresponding to the radial velocities of the two lines of the same resonance doublet and also of the intercombination lines of the same ion as well as the usually small values of the $\mathrm{C}$ IV radial velocity mean - mean resonance doublet radial velocities of other ions, would be hard to explain, if effects on radial velocities of the iron forest, which should not be the same for differnt lines, were important.

\subsection{Clues about the nature of the observed radial velocity shifts for different systems}

More clues concerning the nature of the shifts can be obtained from the time variations and line profiles, for which we have information especialy from the observations of $\mathrm{Z}$ And and RW Hya.

The periodic variation of the $\mathrm{Z}$ And $\mathrm{C}$ IV resonance doubletintercombination line radial velocity means suggests a maximum at phases not long after conjunctions when the cool component is nearer the observer. This might be understood if the increased redshift of $\mathrm{Z}$ And at such phases is due to larger cool component wind line absorption on the short wavelength side of resonance line emission for strongly ionised atoms, with perhaps additional effects behind the cool giant at that phase. Among such effects there is wind focussing towards the orbital plane, with a three dimensional spiral stream, which occurs when the cool component does not quite fill its Roche lobe (Gawryszczak et al. 2003). This interpretation is not contradicted by the C IV and N v fluxes found by Fernandez-Castro et al. (1988), including a minimum near phase zero, confirmed by later more numerous observations. However, Fernandez-Castro et al. (1988) also find simlar minima for the intercombnation lines, so something else than line absorption is also involved.

There has been a certain amount of disagreement and uncertainty about the causes of the outbursts of $\mathrm{Z}$ And and similar symbiotic systems. Our ultraviolet line study may be relevant to this question. According to Sokoloski et al. (2006), based on later observations after the end of the life of IUE, outbursts of $\mathrm{Z}$ And can be understood as disk instabilites, which in the case of major outbursts are followed by thermonuclear burning. The $V$ magnitude of one outburst, studied by these authors, became 


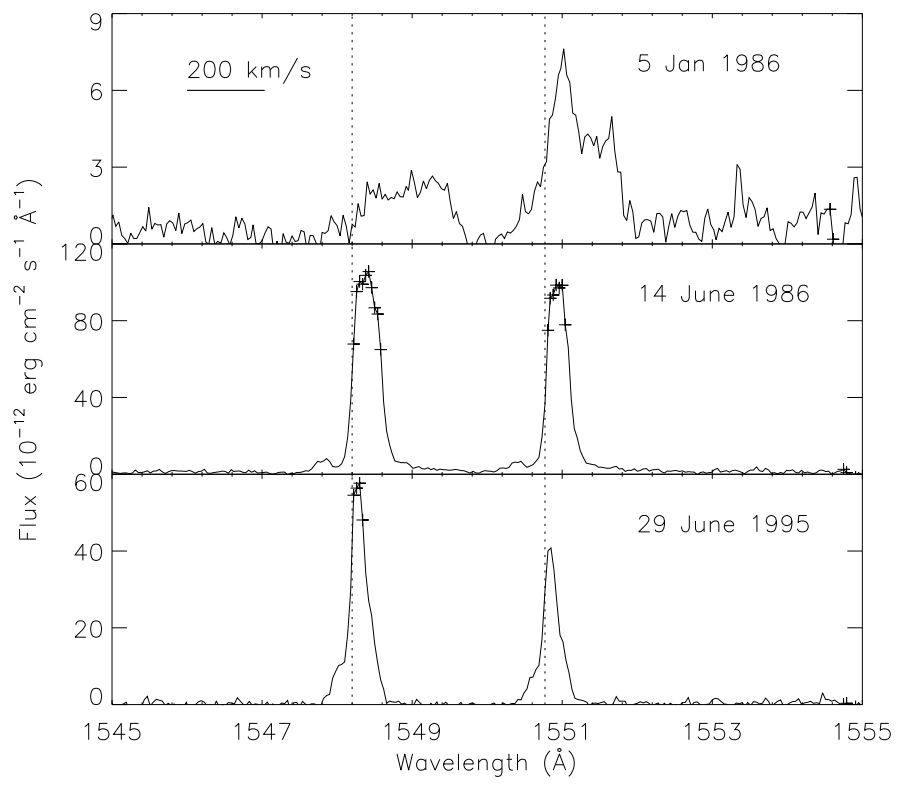

Fig. 10. CIV doublet profiles of $\mathrm{Z}$ And for 3 dates. The upper panel is for phase 0.960 during a major outburst, the middle panel for phase 0.178 at a fainter stage of outburst and the lower panel for phase 0.523 in quiescence.

brighter than about 9.7, when according to them thermonuclear burning started. Bisikalo et al. (2006) also suggested an increase in thermo-nuclear burning during outbursts as well as effects of colliding winds. They made fairly detailed calculations.

Figure 10 shows the IUE profiles of the C IV resonance doublet of $\mathrm{Z}$ And at three epochs. The first in the upper panel is at phase 0.961 during a major outburst, when according to Sokoloski et al. (2006) thermonuclear burning could have occured. On this date the line profiles were faint and complex so we do not attempt to give radial velocities in Table 1 . The middle panel shows the profiles at phase 0.171 , in a fainter stage of outburst, while the bottom panel shows the profiles at phase 0.523 in quiescence. The greater width of lines in outburst, previously detected by Fernandez-Castro et al. (1995), is clearly seen through the presence of very weak wings in the fainter stage of outburst and is gigantic during what could be a thermo-nuclear burning stage according to Sokoloski et al. Figure 10 shows particularly a large quantity of redshifted emission. However we can note in addition to faint red wings, the presence of what looks like blue shifted absorption superposed on weak line emission in the fainter stage of the outburst. Fernandez-Castro et al. (1995) suggested that envelopes were ejected during outburst; another interpretation is discussed below.

We may try to understand the changing profiles, shown in Fig. 10, as due to a decrease in ionising radiation near the maximum of outburst, corresponding to a lower photospheric temperature of an expanded white dwarf. In addition we expect the optical thickness of the C IV resonance lines to be probably very large; because taking an electron density of the order of $10^{10} \mathrm{~cm}^{-3}$ from Altamore et al. (1981) and from FernandezCastro et al. (1988), a solar abundance of carbon, and assuming 10 percent of carbon three times ionised, would lead to the weaker line becoming optically thick for distances of only $2 \times 10^{9} \mathrm{~cm}$. That is small compared with the binary separation times the sine of the inclination of $7 \times 10^{12} \mathrm{~cm}$ of Mikolajewwska (2003). The fractional abundance of carbon in the form of $\mathrm{C}^{+++}$ used, is of the order of that calcuated for different conditions by Nussbaumer (1982). Therefore, the enlargement of what is still visible of the C IV ion doublet lines towards the red in very probable conditions of large optical thicknesses near the cool component at that time, might be due to radiative transfer in an expanding medium such as the cool component wind or due to a region of collision between the winds from the components rather than due to an optically thinner ejected shell. Iron forest absorption can be expected to also play a role in the profile. An explanation only involving line emission due to the wind from the hot component is unlikely, because the escape velocity from the photosphere of the outbursting component would for a largest measured radius of 0.36 solar radii and a white dwarf mass of 0.65 solar masses (taking the estimates of Sokoloski et al. 2006) be then around $830 \mathrm{~km} \mathrm{~s}^{-1}$. A wind from that component might be expected to be probably faster, while in outburst the red sides of those resonance line profiles, which are unaffected by any classical P Cygni absorption, do not extend to more than about $300 \mathrm{~km} \mathrm{~s}^{-1}$.

We may note in this connection that Lamers et al. (1995), in their study of wind terminal velocities of hot stars near the main sequence, found ratios of the terminal to escape velocity, decreasing from around 2.7 at an effective temperature of more than $40000 \mathrm{~K}$ and highly ionized winds to 0.7 at an effective temperature of $8000 \mathrm{~K}$ and much less ionized winds, which produce P Cygni spectral line profiles of $\mathrm{C}$ II, $\mathrm{Al}$ III and $\mathrm{Mg}$ II. In addition Dumm et al. (2000) state that the terminal velocities of central stars of planetary nebulae are typically 2.5 times the escape velocity. Let it be noted however that the hot component wind could be concentrated towards the polar axis by the white dwarf magnetic field and/or the accretion disk, so the observed radial velocity would be then the above expected value times the cosine of the inclination and so be smaller.

We may also note that Sokoloski et al. (2006) observed P Cygni profiles for Z And in outburst in the far ultraviolet, using the FUSE satellite. An enhanced continuum was seen by them during outburst, classical P Cygni blueshifted absorption being strong at phases 0.116 to 0.155 , when the continuum was also strong. P V $1117 \AA$ absorption, indicating the presence of a not very fast wind, is clearly visible to about $-270 \mathrm{~km} \mathrm{~s}^{-1}$ at phase 0.116 in their Fig. 6.

We can compare our AG Dra results with results given in previous papers for that system. Let us note that Viotti et al. (1984) found that the N V lines were broader when the luminosity of AG Dra was higher like for Z And. These authors found in addition P Cygni profiles for all epochs of N V $1238 \AA$ with a terminal velocity of $170 \mathrm{~km} \mathrm{~s}^{-1}$ for the stellar wind supposed to produce it and line assymmetry suggesting $\mathrm{P}$ Cygni absorption of line emission, which was also present when the continuum was weak. Young et al. (2005) observed AG Dra with FUSE and saw a redshift of the O VI resonance doublet (which had a $\mathrm{P}$ Cygni profile) relative to the intercombination $\mathrm{Ne} \mathrm{V}$ and $\mathrm{Ne} \mathrm{VI}$ lines. However their measurements indicated no redshift for the S IV and S VI zero energy lines. These last mentioned authors suggest that the O VI P Cygni absorption is only that of the resonance doublet, superposed on a false continuum, produced by electron scattering wings. We may finally note that the geometry of this system with a fairly small preferred orbital inclintion of $30-45^{\circ}$ according to Mikołajewska et al. (1995) as well as a low metal abundance, may play roles in reducing shifts between the resonance and intercombination lines.

We shall here just emphasize one of the results for SY Mus. The constancy of the CIV radial velocity mean at different orbital phases reminds us of the resonance line mean constant 


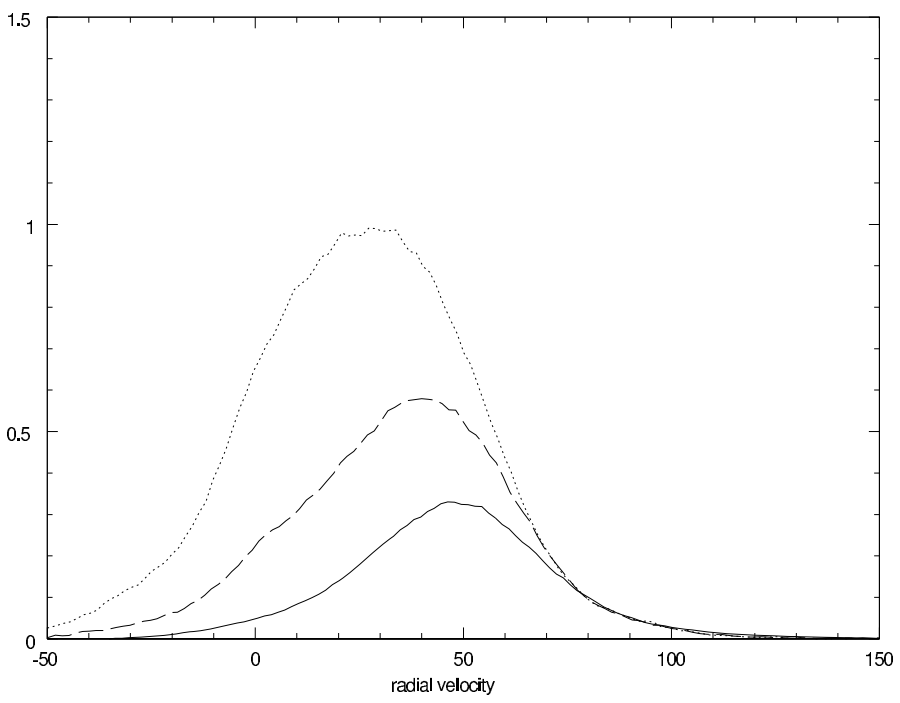

Fig. 11. HST/STIS profiles for RW Hya on MJD 52040 (phase 0.825). The full line shows the C IV $1551 \AA$ profile, the dotted line the N IV] $1486 \AA$ profile and the dashed line the O IV $1401 \AA$ profile

velocity, found by Mikołajewska et al. (2006) for CI Cyg, which like SY Mus is a high inclination eclipsing system. Unlike in the case of CI Cyg, we do not have very high resolution HST spectra for SY Mus, but we may be able to invoke a similar explanation to that given by Mikołajewska, Friedjung and Quiroga, explaing the redshift of the resonance doublet emission lines by the presence of a circum-binary region. Such a region could be near the plane of the orbit.

As far as RW Hya is concerned, the observations available, do not enable us to say anything certain about the variation of redshift with orbital phase. According to Dumm et al. (2000), low resolution UV spectra indicate a flux decrease in the continuum between $1250 \AA$ and $1290 \AA$ at phase 0.78 relative to phase 0.71 , which was almost over at phase 0.81 . They interpreted this result as due to additional apparent extinction by Rayleigh scattering of neutral hydrogen and due to iron forest absorption in an accretion wake produced by wind accretion. Our examination indicates no large variation in the values of C IV-intercombination line radial velocity between phases in the range 0.749 to 0.825 on the high resolution HST/STIS spectra. In any case, there is no accretion wake, if accretion is by Roche lobe overflow, as suggested by the observations of ellipsoidal light variability in the near infrared according to Rutkowski et al. (2007) and another explanation is required for the observations of ultraviolet fluxes in such a case than that of Dumm et al. (2000). Another possibility is absorption due to impact of the stream. In addition Dumm et al. (1999) suggest that the density distribution around the M giant of SY Mus is assymetric with additional extinction than that due to Rayleigh scattering, which they suggest is produced by the iron forest.

Unlike most of the spectra studied here, those of RW Hya did have a significant continuum, with P Cygni absorption components superposed on it, which could have contributed to some measured velocities of the N V and Si IV lines. The edge velocities relative to the systemic velocity are lower than $200 \mathrm{~km} \mathrm{~s}^{-1}$. Let us note also the presence of broad wings of more than $20 \AA$ wide around the CIV resonance lines. It remains to be seen whether that can be explained by electron scattering.

The high resolution HST/STIS spectra of RW Hya can be used to look at the line profiles, in order to better understand the radial velocity shifts. Figure 11 shows profiles of the C IV $1551 \AA$, N IV] $1486 \AA$ and O IV] $1401 \AA$ lines. The weak continuum fluxes of less than $2 \%$ the line centre fluxes have been subtracted, the profiles being insensitive to the continuum and unaffected by the iron forest absorption. The C IV profile was divided by 3 and the O IV] profile by 1.7, so as to make their red wings coincide approximately. One sees that the blue wings of the C IV and O IV] lines are reduced with respect to N IV]. The C IV line is optically thick, so the reduction appears to be due to P Cygni absorption of line emission, starting at radial velocities which are positive relative to the systemic velocity of 12.4 or $12.9 \mathrm{~km} \mathrm{~s}^{-1}$ (both values being given as alternatives by Belczynski et al. 2000 and Mikolajeswska 2003) and a cool giant radial velocity $7.8 \mathrm{~km} \mathrm{~s}^{-1}$ smaller than those values at phase 0.825 . This could suggest the presence of a line absorbing wind from the cool component absorbing some of the line emission coming from regions rotating not very rapidly around the white dwarf with perhaps a contribution to the absorption at certain orbital phases by a stream from the inner Lagrangian point. The latter is possible for high inclination eclipsing systems like RW Hya if the cool component fills its Roche lobe (Rutkowski et al. 2007). Let us note that the wind from the cool component can be deviated by the gravitational field of the compact component. The O IV] line is optically thin, so no explanation, invovlving absorption can work for this line. The effect may be explainable by a large part of the intercombination emission line flux coming from the already mentioned wind from the cool component in front of an accretion disk, with the latter being opticaly thick in the continuum, so occulting emission behind it, as well as a possible contribution to line emission due to the presence of the already mentioned stream from the inner Lagrangian point in front of the disk. Such effects might produce other emission line shifts. Let us finally note that it is not quite clear to what extent the small absolute redshift of the N IV] line centre relative to the systemic and cool giant velocities is real. In any case the very incomplete phasing of the HST/STIS spectra makes it hard to draw more conclusions.

\section{Conclusions}

A number of new results have been obtained, from a more detailed analysis of relative emission line shifts, which conclude the study of suitable ultraviolet spectra, which are available.

The relative shift between the radial velocities of the resonance lines and the intercombination lines of multiply ionised atoms is confirmed for most systems and appears to be not due to various artifacts. It is rather probably due to the absorption component of P Cygni profiles of the cool component's wind, which must in many cases be able to absorb emission from the emission part of line profiles and not only radiation from the continuous spectrum. That places strong constraints on the geometry, which we might try to interpret as for instance involving absorption of line radiation from near the outer edge of an accretion disk. The variation of the shifts with orbital phase, especialy for $\mathrm{Z}$ And, can be understood as due to a greater optical thickness of regions connected with the cool component's wind on its far side with respect to the compact component. However the wide redshifted profile of $\mathrm{C}$ IV of $\mathrm{Z}$ And near phase zero during outburst may still be due to a radiative transfer effect.

Ionisation potential dependent stratification of radial velocity is present for the intercombination lines, O IV] being much more redshifted than intercombination lines of less ionised atoms. This could be connected with occultation by an accretion disk, which is optically thick in the continuum. However let us note 
that our data do not enable us to be certain about any difference for Z And and AG Dra in outburst, if a comparison is made with quiescence We could expect that as a change in the properties of an accretion disk might be expected.

Differences can be due to different geometries and metal abundances. AG Dra in particular has a strong metal underabundance. Streams and cool component winds affected by the presence of the hot component, may play a major role. However the sample of symbiotic systems with high spectral resolution ultraviolet observations at many different orbital phases is very small, making it difficult to look for correlations with the properties of these systems. It is therefore probably not convenient to make more detailed speculations at the present time.

Acknowledgements. This research has been partly supported by Polish research grants 1P03D 01727 and N203 395539, and by the European Associated Laboratory "Astrophysics-Poland-France". It also made use of the NASA Astrophysics Data System and SIMBAD database. We thank Peter Young for giving information on line profiles and other possible channels of possible pumping of excited Fe II levels in AG Dra. J. Zorec must also be thanked for supplying a computer programme, while a friend helped in the preparation of one figure.

\section{References}

Altamore, A., Baratta, G. B., Cassatella, A., et al. 1981, ApJ, 245, 630 Belczyński, K., Mikołajewska, J., Munari, U., Ivison, R. J., \& Friedjung, M. 2000, A\&AS, 146, 407

Bisikalo, D. V., Boyarchuk, A. A., Kilpio, E. Yu., Tomov, N. A., \& Tomova, M. T. 2006, Astron. Rep., 50, 722

Clegg, R. E. S., Miller, S., Storey, P. J., et al. 1999, A\&AS, 135, 359

Dumm, T., Schmutz, W., Schild, H., et al. 1999, A\&A, 349, 169
Dumm, T., Folini, D., Nussbaumer, H., et al. 2000, A\&A, 354, 1014 Eriksson, M., Johansson, S., \& Wahlgren, G. M. 2006, A\&A, 451, 157 Fekel, F. C., Hinckle, K. H., Joyce, R. R., et al. 2000, AJ, 120, 3255

Fernández-Castro, T., Cassatella, A., Giménez, A., et al. 1988, ApJ, 324, 1016 Fernández-Castro, T., González-Riestra, R., Cassatella, A., Taylor, A. R., \& Seaquist, E. R. 1995, ApJ, 442, 366

Friedjung, M., Stencel, R. E., \& Viotti, R. 1983, A\&A, 126, 407

Friedjung, M., Gális, R., Hric, L., et al. 2003, A\&A, 400, 595

Gawryszczak, A. J., Mikołajewska, J., \& Różyczka, 2003, A\&A, 398, 159

Kenyon, S. J., Oliverson, N. A., Mikołajewska, J., et al. 1991, AJ, 101, 637

Lamers, H. J. G. L. M., Snow, T. P., \& Lindholm, D. M. 1995, ApJ, 455, 269

Mikołajewska, J. 2003, in Symbiotic Stars Probing Stellar Evolution, ed. R. L. M. Corradi, J. Mikołajewska, \& T. J. Mahony, PASP Conf. Ser., 303,9

Mikołajewska, J. 2007, Baltic Astron., 16, 1

Mikołajewska, J., \& Kenyon, S. J. 1996, AJ, 112, 1659

Mikołajewska, J., Kenyon, S. J., Mikołajewski, M., Garcia, M. R., \& Polidan, R. S. 1995, AJ, 109, 1289

Mikołajewska, J., Friedjung, M., \& Quiroga, C. 2006, A\&A, 460, 191

Nussbauner, H. 1982, in The Nature of Symbiotic Stars, ed. M. Friedjung, \& R. Viotti, Ap\&SS Library, 95, (Reidel), 85

Nussbaumer, H., Schild, H., Schmid, H. M., et al. 1988, A\&A, 198, 179

Patriarchi, P., Morbidelli, L., Perinotto, M., et al. 2001, A\&A, 372, 644

Penny, L. R., Ouzts, C., \& Gies, D. R. 2008, ApJ, 681, 554

Shore, S. N., \& Aufdenberg, J. P. 1993, ApJ, 416, 355

Rutkowski, R., Mikołajewska, J., \& Whitelock, P. A. 2007, Baltic Astron., 16, 49

Sokoloski, J. L., Kenyon, S. J., Espey, B. R., et al. 2006, ApJ, 636, 1002

Schmutz, W., Schild, H., Mürset, U., et al. 1994, A\&A, 288, 819

Viotti, R., Altamore, A., Baratta, G. B. Cassatella, A., \& Friedjung, M. 1984, ApJ, 283, 226

Young, P. R., Dupree, A. K., Espey, B. R., Kenyon, S. J., \& Ake, T. B. 2005, ApJ, 618, 891

Young, P. R., Dupree, A. K., Espey, B. R., et al. 2006, ApJ, 650, 1091 